\title{
OPEN All-cause mortality of patients with idiopathic pulmonary fibrosis: a nationwide population-based cohort study in Korea
}

\author{
Sung Jun $\mathrm{Ko}^{1,4}$, Sun Mi Choi ${ }^{2,4}$, Kyung-Do Han ${ }^{3}$, Chang-Hoon Lee ${ }^{2}$ \& Jinwoo Lee ${ }^{2 \bowtie}$
}

Most epidemiologic studies of patients with idiopathic pulmonary fibrosis (IPF) have been conducted in North America and Europe. Moreover, there are limited data concerning the cause of death and cause-specific mortality rate of IPF patients in population-based studies. We analyzed information from the Korean National Health Insurance Service database from 2006 to 2016. Patients with a diagnosis code of IPF were extracted from the database and we included those who satisfied the narrow definition of IPF diagnosis. Age- and sex-matched controls were randomly selected at a case-to-control rate of 1:3. We included 42,777 patients newly diagnosed with IPF during the study period. Their mean age was 64.6 years, and $65.4 \%$ were male. The age-standardized mortality rates were 85.66 (95\% confidence interval [Cl] 84.45-86.89) per 1000 person-years. The survival rates of IPF patients $1,2,3,5$, and 10 years after IPF diagnosis were $84.5 \%, 77.4 \%, 71.9 \%, 62.9 \%$, and $48.4 \%$, respectively. The standardized mortality ratio of IPF patients compared to that of the normal population was 4.66. The leading cause of death in IPF patients was respiratory causes, followed by cancer. Patients with IPF in Korea showed significantly higher mortality compared to the general population.

Idiopathic pulmonary fibrosis (IPF) is the most common idiopathic interstitial pneumonia and is associated with a high mortality rate ${ }^{1}$. While there is evidence of increasing IPF incidence and mortality ${ }^{2-4}$, most epidemiologic data on IPF patients, including mortality, were derived from patients in Europe and North America ${ }^{5}$ and the results are highly variable according to study design and statistical methods used to estimate mortality ${ }^{2-4,6-12}$. The mortality of IPF patients is also variable between ethnicities ${ }^{2,6,9}$. Moreover, the reported mortality of IPF patients in population-based studies has not been compared to that of the general population.

In East Asia, epidemiological data on IPF based on a nationwide population database are scarce. Although two population-based studies were performed in Taiwan and Japan ${ }^{11,12}$, the sample sizes were much smaller than those of studies from Europe and North America. Cohort studies of IPF patients in Korea have investigated the clinical course of IPF; however, such studies may be prone to selection bias and only included a small number of participants and, thus, lack generalizability ${ }^{13,14}$.

Despite the high mortality of IPF patients, there are limited data concerning the causes of death and causespecific mortality rate of IPF patients in population-based studies ${ }^{2,6,11}$. Along with differences in the mortality rates of IPF patients, there may also be differences in the causes of death across different ethnicities. While the leading cause of death among IPF patients was respiratory causes, followed by ischemic heart disease in the United States ${ }^{2,6}$, the second most frequent cause of death was lung cancer in Japan ${ }^{11}$. To our knowledge, causespecific mortality rates of IPF patients in population-based studies have not been compared to those of the general population.

One of the main causes of death in IPF patients is respiratory failure due to disease progression which is often inevitable despite advanced care. Except for IPF patients awaiting lung transplantation, mechanical ventilator support for respiratory failure in IPF patients is generally not recommended because of their very high mortality ${ }^{15-18}$. However, the exact proportion of patients with IPF receiving intensive care before their deaths is unknown.

${ }^{1}$ Department of Internal Medicine, Wonkwang University Sanbon Hospital, Gunpo, Republic of Korea. ${ }^{2}$ Division of Pulmonary and Critical Care Medicine, Department of Internal Medicine, Seoul National University College of Medicine, Seoul National University Hospital, 101 Daehak-Ro, Jongno-Gu, Seoul 03080, Republic of Korea. ${ }^{3}$ Department of Biostatistics, College of Medicine, The Catholic University of Korea, Seoul, Republic of Korea. ${ }^{4}$ These authors contributed equally: Sung Jun Ko and Sun Mi Choi. ${ }^{\bowtie}$ email: realrain7@gmail.com 
This study investigated the all-cause and cause-specific mortality rates of IPF patients and compared them to the age- and sex-matched general population using a nationwide population-based database. The causes of death and rates of intensive care unit (ICU) admission before death were also assessed.

\section{Methods}

Study participants. The medical demands of nearly all Korean citizens are mandatorily covered by the National Health Insurance Service (NHIS). From the beginning of full coverage in 1989, the NHIS in Korea has accumulated a tremendous amount of data, including patient diagnosis codes and their claims for medical tests and prescriptions. The NHIS has provided its data to medical researchers for the public interest since the establishment of the database in 2002. A detailed description of the NHIS database has been published elsewhere ${ }^{19}$.

A more narrow definition of IPF was used in this study. Patients with the diagnosis code of IPF (Korean Standard Classification of Disease code J841 or J8418) from January 2006 to December 2016 were initially selected for inclusion in the present study. A narrow definition of IPF was fulfilled after excluding these three conditions. (1) Since the diagnosis of IPF requires multidisciplinary discussion, we excluded patients if the diagnosis of IPF was claimed only in the primary clinics but not in referral hospitals. (2) Patients who did not undergo a pulmonary function test (PFT) within 6 months and chest computed tomography (CT) within 1 year of IPF diagnosis also were excluded. (3) Patients with diagnosis codes of connective tissue diseases (CTDs) were also excluded by their diagnostic codes of CTDs to exclude CTD-associated interstitial lung diseases. To compare the mortality of IPF patients with that of the general population without IPF, age- and sex-matched controls (three times the number of cases) were randomly extracted from the NHIS database. The comorbidities of the patients in the IPF and control groups were collected from their diagnosis codes. Household income and place of residence were also gathered for comparisons between groups. Death certificate data were obtained from the National Statistical Office of Korea to evaluate the survival and causes of death of the study participants.

Claims data of patients admitted to the ICU within 30 days before the date of death were collected. These patients were considered as having received end-of-life care in the ICU.

This study was approved by the Institutional Review Board (IRB) of the Seoul National University Hospital (SNUH) (IRB No. E-1804-046-936). The requirement for informed consent was waived by the IRB of SNUH due to the retrospective design of this study utilizing the NHIS database. All research was performed in accordance with the Declaration of Helsinki concerning the ethical principles for medical research.

Statistical analysis. The mortality rate was described as the incidence rate per 1000 person-years. Mortality rates were calculated in both male and female individuals in the IPF and control groups. The hazard ratio (HR) of IPF patients compared to that in the control group adjusted for age, sex, presence of comorbidities (ischemic heart disease, stroke, cerebrovascular disease, cancer, diabetes mellitus, hypertension, dyslipidemia, and liver disease), household income, and place of residence was analyzed by Cox regression analysis. The differences in cause-specific mortality rates between IPF patients and the controls were also analyzed. We also evaluated the difference between all-cause mortality of IPF patients and the control group using Kaplan-Meier curve analysis.

To calculate the standardized mortality ratio (SMR) of IPF patients, we compared the observed death events in IPF patients to the expected death events of the general population derived from Korean Statistical Information Service data. We also calculated the SMR in the pre-stratified age groups.

Statistical analysis was performed using R (version 3.2.3, https://www.R-project.org/). All statistical tests were two-sided and differences with $p<0.05$ were considered statistically significant.

\section{Results}

Study population. From January 2006 to December 2016, 58,931 patients were identified in the NHIS database as having been diagnosed with IPF at secondary or tertiary referral hospitals. The date of IPF diagnosis was defined as the index date of the IPF patients and they were followed thereafter. Patients who did not undergo a pulmonary function test within 6 months or chest CT scan within 1 year of the index date (7150 patients) were excluded from the analysis. Patients who were diagnosed with CTD (6076 patients) were also excluded. The remaining 45,705 patients with IPF were matched with controls according to age and sex at a 1:3 ratio. After matching, 2928 patients with IPF whose matched controls died before the index date were additionally excluded. Finally, this study included 42,777 patients diagnosed with IPF and a 1:3 control group of 128,331 people (Fig. 1).

Baseline characteristics. The mean age of the IPF patients was $64.6 \pm 13.8$ years, and $65.4 \%$ were male. Compared to the control group, patients in the IPF group more often had ischemic heart disease, cerebrovascular disease, cancer, diabetes mellitus, hypertension, dyslipidemia, and chronic liver disease. Household income and place of residence did not differ between the two groups (Table 1).

All-cause mortality rate of patients with IPF. The all-cause mortality rate of the patients with IPF was 85.663 per 1000 person-years, which was approximately three times higher than that of the control group $(29.525$ per 1000 person-years, $p<0.001)$. The mortality rate of male IPF patients was 97.617 per 1000 personyears, which was significantly higher than that of female IPF patients $(65.895$ per 1000 person-years, $p<0.001)$. The HR of mortality in patients with IPF compared to that in the control group was 2.918 after adjusting for age, sex, presence of comorbidities, household income, and place of residence. The adjusted HRs of male and female IPF patients compared to the control group were 2.885 and 2.989 , respectively (Table 2).

The survival rates of IPF patients were $84.5 \%, 77.4 \%, 71.9 \%, 62.9 \%$, and $48.4 \%$ at $1,2,3,5$, and 10 years of follow-up, respectively. Kaplan-Meier curves of male and female IPF patients and their controls are shown in Fig. 2. 


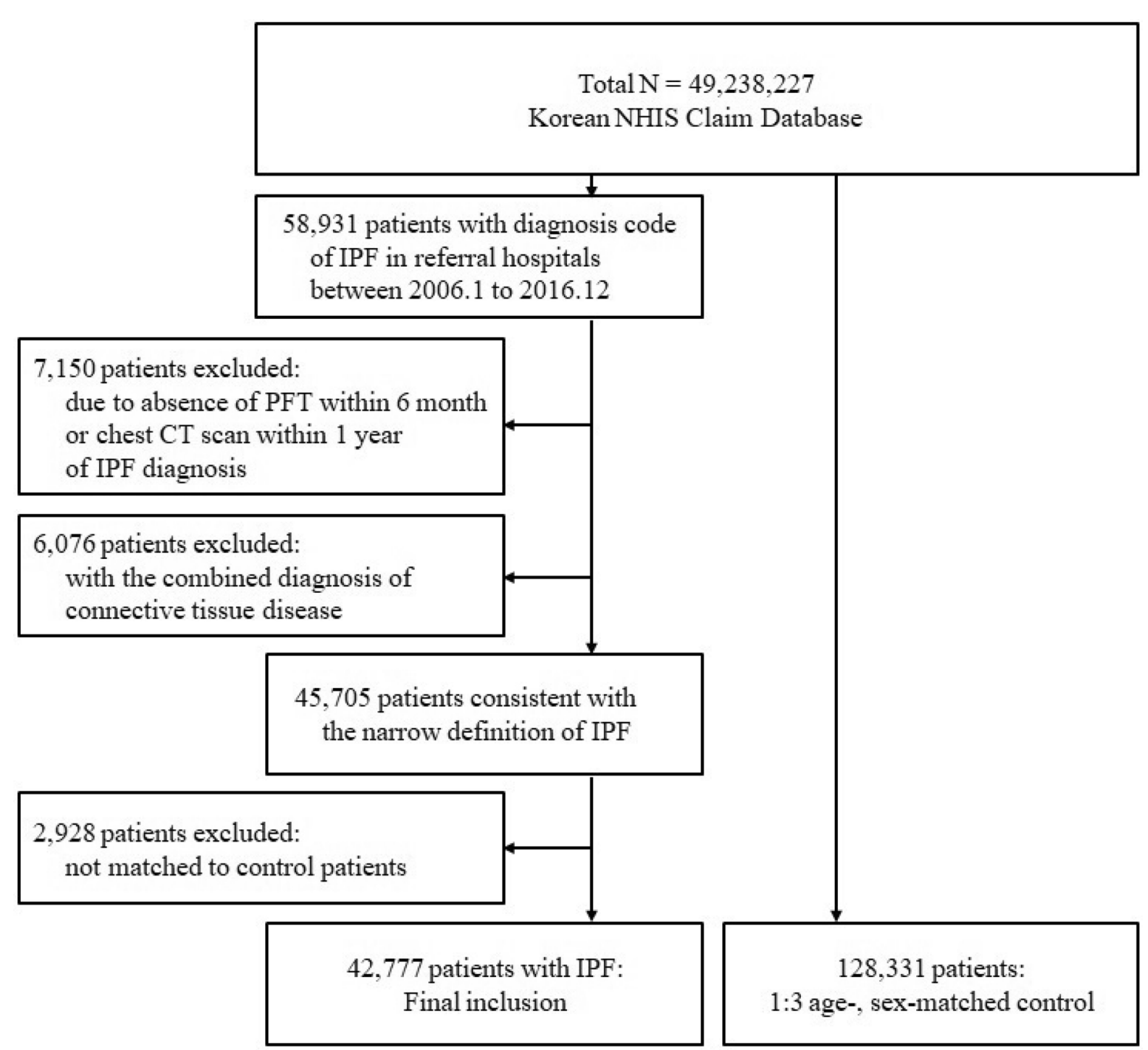

Figure 1. Inclusion flow chart. NHIS National Health Insurance Service, IPF idiopathic pulmonary fibrosis, PFT pulmonary function test, CT computed tomography.

Cause-specific mortality rate of patients with IPF. The leading cause of death in IPF patients was respiratory causes, followed by cancer. Among IPF patients, $37.8 \%(7417$ of 18,905$)$ died due to respiratory causes, which was about ten times higher than that in the control group (adjusted HR 9.743, $p<0.001$ ). Patients with IPF also showed higher risks of death from cancer, cardiovascular cause, and suicide compared to the control group (adjusted HR 3.007, 1.739, and 2.085, respectively; $p<0.001$ ). Respiratory causes of death were more frequently observed in females and cancer was more frequently observed in males (Table 2).

Standardized mortality ratio of IPF patients. Compared to the expected mortality rate of the general population, the SMR of IPF patients was 4.659 (95\% confidence interval 4.593-4.726). Although the mortality rate was higher in the older age group than that in the younger age group, the SMR was lower in the older age group, except for that in patients in their $50 \mathrm{~s}$, which was slightly higher than the SMR in the $40 \mathrm{~s}$ in both sexes (Table 3).

ICU admission before death in patients with IPF. Among the deceased IPF patients, $13.8 \%$ were admitted to the ICU before death. The proportion of patients with ICU admission before death was higher in male patients than in female patients $(14.2 \%$ vs. $12.7 \%, p=0.006)$.

\section{Discussion}

This nationwide general population-based study showed that Korean IPF patients had significantly higher allcause and cause-specific mortality rates compared to those in the control group without IPF. To our knowledge, this is the first nationwide, large-scale, general population-based study on this subject in Asia. Previous two studies from Japan and Taiwan were either with only a small number of 553 IPF patients ${ }^{11}$ or used a database 


\begin{tabular}{|c|c|c|c|}
\hline & IPF group & Control group & $p$ value \\
\hline Number of participants (n) & 42,777 & 128,331 & \\
\hline Age, mean $\pm \mathrm{SD}$ (years) & $64.6 \pm 13.8$ & $64.6 \pm 13.8$ & $>0.999$ \\
\hline Male sex, n (\%) & $27,971(65.4)$ & $83,913(65.4)$ & $>0.999$ \\
\hline \multicolumn{4}{|l|}{ Comorbidities } \\
\hline Ischemic heart disease, $\mathrm{n}(\%)$ & $11,592(27.1)$ & $14,853(11.6)$ & $<0.001$ \\
\hline Cerebrovascular disease, $\mathrm{n}(\%)$ & $19,342(45.2)$ & $51,494(40.1)$ & $<0.001$ \\
\hline Cancer, n (\%) & $5246(12.3)$ & $5678(4.4)$ & $<0.001$ \\
\hline Diabetes mellitus, $\mathrm{n}(\%)$ & $8655(20.2)$ & $18,303(14.3)$ & $<0.001$ \\
\hline Hypertension, $\mathrm{n}(\%)$ & $17,530(41.0)$ & $48,857(38.1)$ & $<0.001$ \\
\hline Dyslipidemia, n (\%) & $9676(22.6)$ & $21,930(17.1)$ & $<0.001$ \\
\hline Chronic liver disease, $\mathrm{n}(\%)$ & $11,033(25.8)$ & $25,305(19.7)$ & $<0.001$ \\
\hline Household income, n (\%) & & & 0.259 \\
\hline 1st quintile & $10,623(24.8)$ & $31,017(24.2)$ & \\
\hline 2nd quintile & $6547(15.3)$ & $20,269(15.8)$ & \\
\hline 3rd quintile & $7078(16.6)$ & $21,413(16.7)$ & \\
\hline 4th quintile & 8455 (19.8) & $25,313(19.7)$ & \\
\hline 5th quintile & $10,074(23.6)$ & $30,319(23.6)$ & \\
\hline Place of residence, $n(\%)$ & & & 0.207 \\
\hline Urban & $23,692(55.4)$ & $71,525(55.7)$ & \\
\hline Rural & $19,085(44.6)$ & $56,806(44.3)$ & \\
\hline
\end{tabular}

Table 1. Baseline participant characteristics. IPF idiopathic pulmonary fibrosis, $S D$ standard deviation.

representing only $10 \%$ of all insured Taiwanese patients, and showed a much lower IPF incidence rate ${ }^{12}$ compared to other epidemiologic studies of IPF.

In this study, the survival rates of IPF patients were $84.5 \%, 77.4 \%$, and $71.9 \%$ at 1,2 , and 3 years after diagnosis, respectively. These results are comparable to those of a multicenter cohort study in Korea that reported 1 -, 2-, and 3-year the survival rates of $82.7 \%, 75.7 \%$, and $70.3 \%$, respectively, among 832 IPF patients ${ }^{14}$. A population-based study from Canada reported a survival rate of $79.0 \%, 69.5 \%$, and $63.2 \%$ at 1,2 , and 3 years after diagnosis, respectively ${ }^{7}$. The differences in survival rate are compatible with the results of a recent study in the United States, which observed a lower mortality rate among Asian patients with IPF than that of Caucasian or Hispanic IPF patients ${ }^{6}$.

The all-cause mortality rate of IPF patients was 97.617 per 1000 person-years in men and 65.895 in women in the present study, which was three times higher than that of the control group. Population-based studies reporting the mortality rate of IPF patients consistently showed higher mortality in male patients than that in female patients ${ }^{2-4,6,9}$, except for a study in Brazil that reported similar mortality between sexes ${ }^{20}$. A recent cohort study reported that the presence of cough was associated with higher mortality only in men but not in women ${ }^{21}$; however, the cause of sex differences in IPF mortality remains unknown.

According to the SMR calculated in our study, Korean IPF patients had a 4.7-fold higher risk of mortality than that in the general population. In contrast to the mortality rate of IPF patients which increased with age, the SMR of IPF patients decreased with age. Similar trends have been observed in studies investigating other diseases $^{22,23}$. Although the absolute mortality rate was higher in older patients, the impact of IPF on mortality was higher in younger patients because the expected mortality of the general population at a younger age is very low. To our knowledge, ours is one of the few studies to report the SMR of IPF patients.

Although respiratory causes were the consistent leading cause of death among IPF patients, the proportion of patients who died due to respiratory causes was lower and that of cancer was higher than in reported in previous population-based studies ${ }^{2,6,11}$. As mentioned above, Asian patients with IPF have longer expected survival compared to that in Caucasian patients, which makes them more prone to cancer development during their lives. In Korea, the National Cancer Screening Program allows cost-free screening of stomach, breast, cervix, liver, and colon cancer for almost all Korean citizens ${ }^{24}$. Moreover, the risk of cancer development in patients with IPF is more than twice that in people without $\mathrm{IPF}^{25}$. A significant number of patients diagnosed with cancer through screening may also have been diagnosed with early IPF, which explains the high prevalence of cancer in our study participants. Patients with comorbidities such as IPF may not as receive aggressive cancer treatments as do those without IPF, which might explain the high rate of death by cancer in our study. A cohort study showed higher IPF-specific mortality in females, although the overall mortality was higher in males ${ }^{26}$, which was consistent with our findings.

Among IPF patients who were admitted to the hospital, more than $10 \%$ were mechanically ventilated; however, their mortality rate was sevenfold higher than that of patients not mechanically ventilated, with considerable additional $\operatorname{cost}^{27}$. When mechanically ventilated, the reported mortality rates of IPF patients are as high as $50-94 \%{ }^{17,28,29}$. Thus, the initiation of mechanical ventilation should be recommended in selected IPF patients such as those who are candidates for lung transplantation or patients with reversible causes of respiratory failure ${ }^{18,30}$. In our study, $13.8 \%$ of IPF patients received ICU care before death, and the proportion of patients with ICU 


\begin{tabular}{|c|c|c|c|c|c|c|c|}
\hline & & Total patients (n) & Death (n) & \begin{tabular}{|l|}
$\begin{array}{l}\text { Follow-up duration } \\
\text { (person-year) }\end{array}$ \\
\end{tabular} & $\begin{array}{l}\text { Mortality rate per } \\
1000 \text { person-year }\end{array}$ & \begin{tabular}{|l|} 
aAdjusted HR (95\% \\
CI)
\end{tabular} & $p$ value \\
\hline \multicolumn{8}{|c|}{ Total death } \\
\hline \multirow{2}{*}{ Total } & Control & 128,331 & 25,058 & 848,706 & 29.525 & 1 (Ref.) & \\
\hline & IPF & 42,777 & 18,905 & 220,690 & 85.663 & $2.918(2.861-2.976)$ & $<0.001$ \\
\hline \multirow{2}{*}{ Male } & Control & 83,913 & 18,153 & 543,748 & 33.385 & 1 (Ref.) & \\
\hline & IPF & 27,971 & 13,425 & 137,527 & 97.617 & $2.885(2.819-2.953)$ & $<0.001$ \\
\hline \multirow{2}{*}{ Female } & Control & 44,418 & 6905 & 304,958 & 22.643 & 1 (Ref.) & \\
\hline & IPF & 14,806 & 5480 & 83,162 & 65.895 & $2.989(2.881-3.101)$ & $<0.001$ \\
\hline \multicolumn{8}{|c|}{ Respiratory cause } \\
\hline \multirow{2}{*}{ Total } & Control & 128,331 & $3140(12.5 \%)$ & 848,706 & 3.700 & 1 (Ref.) & \\
\hline & IPF & 42,777 & $7147(37.8 \%)$ & 220,690 & 32.385 & $9.837(9.421-10.271)$ & $<0.001$ \\
\hline \multirow{2}{*}{ Male } & Control & 83,913 & $2451(13.5 \%)$ & 543,748 & 4.508 & 1 (Ref.) & \\
\hline & IPF & 27,971 & $4828(36.0 \%)$ & 137,527 & 35.106 & $8.699(8.273-9.147)$ & $<0.001$ \\
\hline \multirow[b]{2}{*}{ Female } & Control & 44,418 & $689(10.0 \%)$ & 304,958 & 2.259 & 1 (Ref.) & \\
\hline & IPF & 14,806 & $2319(42.3 \%)$ & 83,162 & 27.885 & \begin{tabular}{|l|}
$13.847(12.697-$ \\
$15.101)$
\end{tabular} & $<0.001$ \\
\hline \multicolumn{8}{|l|}{ Cancer } \\
\hline \multirow{2}{*}{ Total } & Control & 128,331 & $6459(25.8 \%)$ & 848,706 & 7.610 & 1 (Ref.) & \\
\hline & IPF & 42,777 & $4528(24.0 \%)$ & 220,690 & 20.518 & $2.381(2.289-2.477)$ & $<0.001$ \\
\hline \multirow{2}{*}{ Male } & Control & 83,913 & $5225(28.8 \%)$ & 543,748 & 9.609 & 1 (Ref.) & \\
\hline & IPF & 27,971 & $3719(27.7 \%)$ & 137,527 & 27.042 & $2.475(2.369-2.586)$ & $<0.001$ \\
\hline \multirow{2}{*}{ Female } & Control & 44,418 & $1234(17.9 \%)$ & 304,958 & 4.046 & 1 (Ref.) & \\
\hline & IPF & 14,806 & $809(14.8 \%)$ & 83,162 & 9.728 & $1.990(1.814-2.183)$ & $<0.001$ \\
\hline \multicolumn{8}{|c|}{ Cardiovascular cause } \\
\hline \multirow{2}{*}{ Total } & Control & 128,331 & $1818(7.3 \%)$ & 848,706 & 2.142 & 1 (Ref.) & \\
\hline & IPF & 42,777 & $690(3.6 \%)$ & 220,690 & 3.127 & $1.415(1.293-1.549)$ & $<0.001$ \\
\hline \multirow{2}{*}{ Male } & Control & 83,913 & $1161(6.4 \%)$ & 543,748 & 2.135 & 1 (Ref.) & \\
\hline & IPF & 27,971 & $449(3.3 \%)$ & 137,527 & 3.265 & $1.448(1.294-1.621)$ & $<0.001$ \\
\hline \multirow{2}{*}{ Female } & Control & 44,418 & $657(9.5 \%)$ & 304,958 & 2.154 & 1 (Ref.) & \\
\hline & IPF & 14,806 & $241(4.4 \%)$ & 83,162 & 2.898 & $1.347(1.157-1.569)$ & $<0.001$ \\
\hline \multicolumn{8}{|l|}{ Suicide } \\
\hline \multirow{2}{*}{ Total } & Control & 128,331 & $511(2.0 \%)$ & 848,706 & 0.602 & 1 (Ref.) & \\
\hline & IPF & 42,777 & $258(1.4 \%)$ & 220,690 & 1.169 & $2.000(1.712-2.335)$ & $<0.001$ \\
\hline \multirow{2}{*}{ Male } & Control & 83,913 & $443(2.4 \%)$ & 543,748 & 0.815 & 1 (Ref.) & \\
\hline & IPF & 27,971 & $212(1.6 \%)$ & 137,527 & 1.542 & $1.940(1.637-2.300)$ & $<0.001$ \\
\hline \multirow{2}{*}{ Female } & Control & 44,418 & $68(1.0 \%)$ & 304,958 & 0.223 & 1 (Ref.) & \\
\hline & \begin{tabular}{|l|} 
IPF \\
\end{tabular} & 14,806 & $46(0.8 \%)$ & 83,162 & 0.553 & $2.355(1.600-3.467)$ & $<0.001$ \\
\hline \multicolumn{8}{|l|}{\begin{tabular}{|l|} 
Others \\
\end{tabular}} \\
\hline \multirow{2}{*}{ Total } & Control & 128,331 & $13,130(52.4 \%)$ & 848,706 & 15.471 & 1 (Ref.) & \\
\hline & IPF & 42,777 & $6282(33.2 \%)$ & 220,690 & 28.465 & $1.861(1.804-1.920)$ & $<0.001$ \\
\hline \multirow{2}{*}{ Male } & Control & 83,913 & $8873(48.9 \%)$ & 543,748 & 16.318 & 1 (Ref.) & \\
\hline & IPF & 27,971 & $4217(31.4 \%)$ & 137,527 & 30.663 & $1.856(1.787-1.928)$ & $<0.001$ \\
\hline \multirow{2}{*}{ Female } & Control & 44,418 & $4257(61.7 \%)$ & 304,958 & 13.959 & 1 (Ref.) & \\
\hline & IPF & 14,806 & $2065(37.7 \%)$ & 83,162 & 24.831 & $1.855(1.757-1.959)$ & $<0.001$ \\
\hline
\end{tabular}

Table 2. All-cause and cause-specific mortality rates of patients with idiopathic pulmonary fibrosis (IPF). IPF idiopathic pulmonary fibrosis, $H R$ hazard ratio, $C I$ confidence interval, Ref reference. ${ }^{a}$ Adjusted by age, sex, ischemic heart disease, stroke, cerebrovascular disease, cancer, diabetes mellitus, hypertension, dyslipidemia, liver disease, household income, and place of residence.

admission was higher in men. Previous studies reported that $18.1 \%$ of the general population of Korea died in the $\mathrm{ICU}^{31}$, compared to $22.4 \%$ of the general population of the United States ${ }^{32}$, with $29.0 \%$ of Medicare beneficiaries receiving ICU care during the last 30 days of their lives ${ }^{33}$. As expected, the proportion of deaths in the ICU of IPF patients in our study was much lower than that reported in the general population.

Our study has several limitations. First, we used the claims data of the NHIS, which limited the diagnostic accuracy of IPF. To overcome this limitation, we used not only the disease code but also several additional criteria as described in the method section. Second, death certificate data were obtained to evaluate the cause of death. Although errors in the cause and/or manner of death recorded on the certificates are reportedly high ${ }^{34}$, the major error is an overrepresentation of nonspecific cardiovascular events as the cause of death ${ }^{35}$. In our study, 
(a) Total

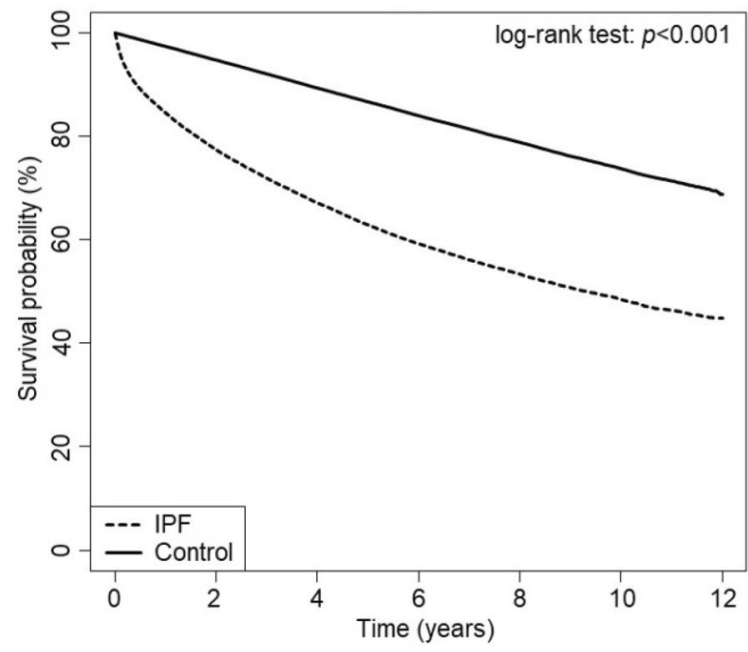

(b) Male and female

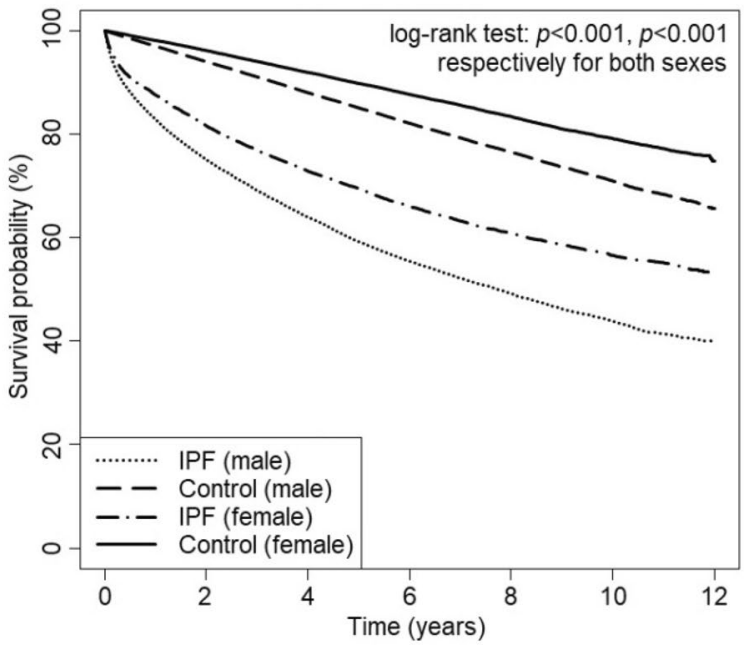

Figure 2. Survival rate of patients with idiopathic pulmonary fibrosis (IPF) compared to control group by Kaplan-Meier curve analysis.

\begin{tabular}{|l|l|c|c|c|c|}
\hline \multicolumn{2}{|c|}{} & Death & Follow-up duration (person-year) & Mortality rate per 1000 person-year & SMR (95\% CI) \\
\hline \multirow{5}{*}{ Total } & 18,905 & 220,690 & 85.7 & $4.659(4.593-4.726)$ \\
\hline \multirow{5}{*}{ Male } & $20-29$ & 40 & 3824 & 65.4 & $15.993(11.037-20.949)$ \\
\cline { 2 - 7 } & $30-39$ & 51 & 6848 & 107.6 & $6.922(5.022-8.822)$ \\
\cline { 2 - 7 } & $40-49$ & 255 & 14,537 & 282.6 & $6.207(5.445-6.968)$ \\
\cline { 2 - 7 } & $50-59$ & 1217 & 27,797 & 622.2 & $7.037(6.641-7.432)$ \\
\cline { 2 - 7 } & $60-69$ & 3933 & 44,924 & 1434.9 & $6.101(5.910-6.292)$ \\
\cline { 2 - 7 } & $70-79$ & 5810 & 32,354 & 3926.1 & $4.574(4.456-4.691)$ \\
\cline { 2 - 7 } & $80-$ & 2119 & 7242 & $11,215.8$ & $2.609(2.498-2.720)$ \\
\hline \multirow{5}{*}{ Female } & $20-29$ & 35 & 2995 & 40.2 & $29.030(19.413-38.648)$ \\
\cline { 2 - 7 } & $30-39$ & 35 & 4888 & 61.6 & $11.622(7.772-15.473)$ \\
\cline { 2 - 7 } & $40-49$ & 112 & 10,740 & 110.6 & $9.427(7.681-11.173)$ \\
\cline { 2 - 7 } & $50-59$ & 325 & 16,291 & 209.6 & $9.518(8.484-10.553)$ \\
\cline { 2 - 7 } & $60-69$ & 995 & 20,947 & 560.7 & $8.471(7.945-8.998)$ \\
\cline { 2 - 7 } & $70-79$ & 2524 & 20,814 & 1978.5 & $6.129(5.890-6.368)$ \\
\cline { 2 - 6 } & $80-$ & 1454 & 6487 & & $2.759(2.617-2.900)$ \\
\hline
\end{tabular}

Table 3. Standardized mortality ratio of patients with idiopathic pulmonary fibrosis (IPF) according to age group. IPF idiopathic pulmonary fibrosis, $S M R$ standardized mortality ratio, CI confidence interval.

the major cause of death was respiratory causes, followed by cancer, with only $3.6 \%$ attributed to cardiovascular causes. Third, we only described the use of ICU facilities within 30 days before the date of death but could not evaluate the reason and appropriateness of ICU admission or whether the reason for ICU admission was correspondent to the cause of death. Fourth, information on the specifics of the cause of death or the presence of acute exacerbation could not be obtained due to the nature of the database.

In conclusion, IPF patients showed a significantly higher mortality rate compared to that in the general population. The leading cause of death in IPF patients was respiratory causes, followed by cancer. The proportion of patients requiring ICU services before death in IPF patients was lower than that in the general population reported in previous studies.

\section{Data availability}

Data are accessible from NHIS database, but the access to data used in this study is only available for the researchers who have applied for and have been granted. Further information is available in online homepage of National Health Insurance Sharing Service (https://nhiss.nhis.or.kr).

Received: 26 February 2021; Accepted: 9 July 2021

Published online: 26 July 2021 


\section{References}

1. Lederer, D. J. \& Martinez, F. J. Idiopathic pulmonary fibrosis. N. Engl. J. Med. 378, 1811-1823 (2018)

2. Olson, A. L. et al. Mortality from pulmonary fibrosis increased in the United States from 1992 to 2003. Am. J. Respir. Crit. Care Med. 176, 277-284 (2007).

3. Hutchinson, J. P., McKeever, T. M., Fogarty, A. W., Navaratnam, V. \& Hubbard, R. B. Increasing global mortality from idiopathic pulmonary fibrosis in the twenty-first century. Ann. Am. Thorac. Soc. 11, 1176-1185 (2014).

4. Navaratnam, V. et al. The rising incidence of idiopathic pulmonary fibrosis in the UK. Thorax 66, 462-467 (2011).

5. Caminati, A., Madotto, F., Cesana, G., Conti, S. \& Harari, S. Epidemiological studies in idiopathic pulmonary fibrosis: Pitfalls in methodologies and data interpretation. Eur. Respir. Rev. 24, 436-444 (2015).

6. Jeganathan, N., Smith, R. A. \& Sathananthan, M. Mortality trends of idiopathic pulmonary fibrosis in the United States from 2004 through 2017. Chest 159, 228-238 (2021)

7. Hopkins, R. B., Burke, N., Fell, C., Dion, G. \& Kolb, M. Epidemiology and survival of idiopathic pulmonary fibrosis from national data in Canada. Eur. Respir. J. 48, 187-195 (2016).

8. Marshall, D. C., Salciccioli, J. D., Shea, B. S. \& Akuthota, P. Trends in mortality from idiopathic pulmonary fibrosis in the European Union: An observational study of the WHO mortality database from 2001-2013. Eur. Respir. J. 51, 1701603 (2018).

9. Mannino, D. M., Etzel, R. A. \& Parrish, R. G. Pulmonary fibrosis deaths in the United States, 1979-1991. An analysis of multiplecause mortality data. Am. J. Respir. Crit. Care Med. 153, 1548-1552 (1996)

10. Raghu, G. et al. Idiopathic pulmonary fibrosis in US Medicare beneficiaries aged 65 years and older: Incidence, prevalence, and survival, 2001-11. Lancet Respir. Med. 2, 566-572 (2014).

11. Natsuizaka, M. et al. Epidemiologic survey of Japanese patients with idiopathic pulmonary fibrosis and investigation of ethnic differences. Am. J. Respir. Crit. Care Med. 190, 773-779 (2014).

12. Lai, C.-C. et al. Idiopathic pulmonary fibrosis in Taiwan-A population-based study. Respir. Med. 106, 1566-1574 (2012).

13. Kim, E. S. et al. Validation of the GAP score in Korean patients with idiopathic pulmonary fibrosis. Chest 147, 430-437 (2015).

14. Lee, S. H. et al. Comparison of CPI and GAP models in patients with idiopathic pulmonary fibrosis: A nationwide cohort study. Sci. Rep. 8, 1-8 (2018).

15. Raghu, G. et al. An official ATS/ERS/JRS/ALAT statement: Idiopathic pulmonary fibrosis: Evidence-based guidelines for diagnosis and management. Am. J. Respir. Crit. Care Med. 183, 788-824 (2011).

16. Collard, H. R. et al. Acute exacerbations of idiopathic pulmonary fibrosis. Am. J. Respir. Crit. Care Med. 176, 636-643 (2007)

17. Mallick, S. Outcome of patients with idiopathic pulmonary fibrosis (IPF) ventilated in intensive care unit. Respir. Med. 102, 1355-1359 (2008)

18. Kondoh, Y., Cottin, V. \& Brown, K. K. Recent lessons learned in the management of acute exacerbation of idiopathic pulmonary fibrosis. Eur. Respir. Rev. 26, 170050 (2017).

19. Lee, J., Lee, J. S., Park, S.-H., Shin, S. A. \& Kim, K. Cohort profile: The national health insurance service-national sample cohort (NHIS-NSC), South Korea. Int. J. Epidemiol. 46, e15(1-8) (2017).

20. Algranti, E., Saito, C. A., Carneiro, A. P. S. \& Bussacos, M. A. Mortality from idiopathic pulmonary fibrosis: A temporal trend analysis in Brazil, 1979-2014. J. Bras. Pneumol. 43, 445-450 (2017).

21. Zaman, T. et al. Differences in clinical characteristics and outcomes between men and women with idiopathic pulmonary fibrosis: A multicenter retrospective cohort study. Chest 158, 245-251 (2021).

22. Lee, E. et al. Mortality and causes of death in patients with atrial fibrillation: A nationwide population-based study. PLoS One 13, e0209687 (2018)

23. Egeland, G. M. et al. Hospitalised patients with unexplained chest pain: Incidence and prognosis. J. Intern. Med. 286, 562-572 (2019).

24. Suh, M. et al. Trends in participation rates for the national cancer screening program in Korea, 2002-2012. Cancer Res. Treat. Off. J. Korean Cancer Assoc. 49, 798-806 (2017).

25. Lee, H. Y., Lee, J., Lee, C., Han, K. \& Choi, S. M. Risk of cancer incidence in patients with idiopathic pulmonary fibrosis: A nationwide cohort study. Respirology 26, 180-187 (2021).

26. Kärkkäinen, M. et al. Underlying and immediate causes of death in patients with idiopathic pulmonary fibrosis. BMC Pulm. Med. 18,69 (2018).

27. Mooney, J. J., Raimundo, K., Chang, E. \& Broder, M. S. Mechanical ventilation in idiopathic pulmonary fibrosis: A nationwide analysis of ventilator use, outcomes, and resource burden. BMC Pulm. Med. 17, 84 (2017).

28. Gaudry, S. et al. Invasive mechanical ventilation in patients with fibrosing interstitial pneumonia. J. Thorac. Cardiovasc. Surg. 147, 47-53 (2014).

29. Rush, B., Wiskar, K., Berger, L. \& Griesdale, D. The use of mechanical ventilation in patients with idiopathic pulmonary fibrosis in the United States: A nationwide retrospective cohort analysis. Respir. Med. 111, 72-76 (2016).

30. Lee, S. H. et al. Korean guidelines for diagnosis and management of interstitial lung diseases: Part 2. Idiopathic pulmonary fibrosis. Tuberc. Respir. Dis. (Seoul) 82, 102-117 (2019).

31. Bae, J. M. et al. The suggestion of social consensus on withdrawing a life-sustaining therapy in terminally ill patients (NECA10A-0003). Korean National Evidence-based Healthcare Collaborating Agency 1-481 (2009). https://www.neca.re.kr/lay1/ program/S1T11C105/conference/view.do?cms_menu_seq=105\&seq=7. Accessed 7 Dec 2020.

32. Angus, D. C. et al. Use of intensive care at the end of life in the United States: An epidemiologic study. Crit. Care Med. 32, 638-643 (2004).

33. Teno, J. M. et al. Site of death, place of care, and health care transitions among US Medicare beneficiaries, 2000-2015. JAMA 320, 264-271 (2018).

34. Brooks, E. G. \& Reed, K. D. Principles and pitfalls: A guide to death certification. Clin. Med. Res. 13, 74-82 (2015).

35. Lakkireddy, D. R. et al. Improving death certificate completion: A trial of two training interventions. J. Gen. Intern. Med. 22, 544-548 (2007).

\section{Acknowledgements}

We would like to thank the staffs of NHIS for providing the claims data of IPF patients and matched controls. We also thank the National Statistical Office of Korea for providing the database from death certificates.

\section{Author contributions}

All authors contributed to the conception and design of the study. K.D.H. had access to all of the data in the study and analyzed it. C.H.L. provided substantial contributions to the interpretation of the data. S.J.K., S.M.C., and J.L. wrote the manuscript. All authors take responsibility for the integrity of the data and the accuracy of the work. All authors have read and approved the final version of the manuscript. 


\section{Competing interests}

The authors declare no competing interests.

\section{Additional information}

Correspondence and requests for materials should be addressed to J.L.

Reprints and permissions information is available at www.nature.com/reprints.

Publisher's note Springer Nature remains neutral with regard to jurisdictional claims in published maps and institutional affiliations.

(c) (1) Open Access This article is licensed under a Creative Commons Attribution 4.0 International License, which permits use, sharing, adaptation, distribution and reproduction in any medium or format, as long as you give appropriate credit to the original author(s) and the source, provide a link to the Creative Commons licence, and indicate if changes were made. The images or other third party material in this article are included in the article's Creative Commons licence, unless indicated otherwise in a credit line to the material. If material is not included in the article's Creative Commons licence and your intended use is not permitted by statutory regulation or exceeds the permitted use, you will need to obtain permission directly from the copyright holder. To view a copy of this licence, visit http://creativecommons.org/licenses/by/4.0/.

(C) The Author(s) 2021, corrected publication 2021 